\title{
Variações interanuais na fenologia de uma comunidade arbórea de floresta semidecídua no sudeste do Brasil
}

\author{
Paulo Rubim ${ }^{1,2,4}$, Henrique E. M. Nascimento ${ }^{3}$ e Leonor Patrícia C. Morellato ${ }^{1}$
}

Recebido em 6/09/2009. Aceito em 15/06//2010

RESUMO - (Variações interanuais na fenologia de uma comunidade arbórea de floresta semidecídua no sudeste do Brasil). As comunidades arbóreas de florestas tropicais localizadas em regiões com clima sazonal tendem a ajustar a fenologia da queda de folhas e da produção de flores e frutos à sazonalidade climática. Neste estudo monitoramos a comunidade arbórea de uma floresta semidecidual no período de quatro anos (2001 a 2004), a fim de analisar a relação entre o número de espécies perdendo folhas, florescendo e frutificando e a variação anual do comprimento do dia, precipitação e temperatura durante o período de observação. Além disso, avaliamos se essas três fenofases repetem os mesmos padrões em anos sucessivos. Regressões lineares simples indicaram que o número de espécies perdendo folhas foi negativamente relacionado com as três variáveis ambientais para os quatro anos de estudo, sendo o comprimento do dia e a temperatura os melhores preditores para esta fenofase. Houve também relação significativa do comprimento do dia com o número de espécies florescendo e frutificando nos quatro anos, porém, precipitação e temperatura não foram relacionadas com estas fenofases em todos os anos de estudo. Os testes de estatística circular indicaram forte sazonalidade para queda foliar, com data média durante o mês de julho independente do ano, ao passo que para floração e frutificação não foi detectada sazonalidade significativa. Como resultado, o número de espécies perdendo folhas foi correlacionado entre todos os anos sucessivos. Entretanto, não houve correlação do número de espécies florescendo e frutificando em 2003 e 2004, provavelmente devido às altas temperaturas ocorridas neste último ano. Os resultados deste estudo sugerem que a queda de folhas é uma fenofase altamente previsível temporalmente. Ao contrário, a floração e a frutificação podem variar ano a ano como conseqüência de anomalias climáticas. Essas variações podem ser relevantes para o entendimento dos efeitos em longo prazo de mudanças climáticas sobre as florestas sazonais.

Palavras-chave: comprimento do dia, floresta Atlântica, mudanças climáticas, sazonalidade

ABSTRACT - (Interannual variation in the phenology of a tree community in a semideciduous seasonal forest in southeast Brazil). Tropical tree communities in regions with seasonal climate usually adjust leaf-fall, flowering and fruiting phenology to climate seasonality. Here we monitored a tree community over a 4-year period in order to assess the relationship between the number of species presenting leaf fall, flower and fruit and annual variation in day length, precipitation and temperature. Moreover, we asked whether the three phenological variables have the same patterns in successive years. Simple regression analyses showed that the number of species shedding leaves was negatively related to the three environmental variables during the four years, with day length and temperature as the best predictors. There was also a relationship between day length and the number of species flowering and fruiting, while rainfall and temperature were only related to these phenophases in some years. The circular statistics indicate highly significant seasonality only for leaf fall, whereas for flowering and fruiting significant seasonal patterns were not detected. The number of species in leaf fall was correlated between all successive years. However, the number of species in flower and fruit was not significantly correlated between 2003 and 2004. Overall, our results suggest that leaf fall is highly predictable over time. On the contrary, flowering and fruiting may present year-to-year variation as a result of climatic anomalies such as the high temperatures detected in 2004. These variations may be relevant to understand the effects of climate change on seasonal forests in long-term studies. Key words: day length, Atlantic forest, climate changes, seasonality

\section{Introdução}

Estudos sobre a fenologia de espécies arbóreas tropicais podem ajudar a entender os efeitos das mudanças climáticas na vegetação, a exemplo do que se tem observado em regiões temperadas (Menzel et al. 2006; Post et al. 2008). Observações fenológicas de longa duração nos trópicos mostram que eventos extremos como El Niño podem afetar a fenologia de plantas (Wright \& Calderon 2006; Zimmerman et al. 2007). Estas observações permitem inferir sobre a possível influência das mudanças do clima nas florestas tropicais, como tem sido sugerido em estudos da dinâmica da Floresta Amazônica (Laurance et al. 2004; Phillips et al. 2004). Portanto, a comparação da variação dos padrões fenológicos de espécies e/ou indivíduos entre anos consecutivos, e sua associação com anomalias climáticas que porventura possam ocorrer no período de estudo (Wright \& Calderon 2006), podem ajudar a compreender a resposta das plantas tropicais às mudanças climáticas. Essas anomalias climáticas deverão acontecer em vários momentos ao longo do tempo de vida de organismos de vida longa e as mudanças nos padrões fenológicos poderá ser uma resposta das espécies arbóreas a curto prazo.

As florestas tropicais localizadas em regiões com marcada sazonalidade climática apresentam eventos fenológicos sazonais, sendo que a queda de folhas, produção de flores e frutos se ajustam à variação anual na precipitação e temperatura (Wright \& van Schaik 1994; Anderson et al. 2005; Stevenson et al. 2008). Fatores evolutivos ou finais, tais como a polinização, herbivoria, predação e dispersão de sementes, germinação e estabelecimento de plântulas, moldariam esses padrões cuja ocorrência estaria limitada pela sazonalidade do clima, além das relações filogenéticas que restringiram a época de manifestação das fenofases em espécies aparentadas (Reich \& Borchert 1984; Rathcke \& Lacey 1985; van Schaik et al. 1993; Boulter et al. 2006; Bolmgren \& Cowan 2008). Portanto, a alternância entre períodos quentes/úmidos e frios/secos pode ser um dos

\footnotetext{
1 Universidade Estadual Paulista, Departamento de Botânica, Laboratório de Fenologia, Grupo de Fenologia e Dispersão de Sementes, Rio Claro, SP, Brasil

2 Universidade Federal de São Carlos, Departamento de Botânica, São Carlos, SP, Brasil

3 Coordenação em Pesquisa em Silvicultura Tropical, INPA, Manaus, Amazonas, Brasil

4 Autor para correspondência: paulo.rubim@yahoo.com.br
} 
principais fatores próximos determinantes dos padrões fenológicos dessas florestas (Alvin \& Alvin 1976; Monasterio \& Sarmiento 1976; Morellato et al. 1989; Reich 1995). Como conseqüência, a maioria das espécies arbóreas das florestas sazonais perdem suas folhas durante a estação mais seca do ano (Leigh \& Windsor 1982; Morellato et al. 1989; Borchert et al. 2002). A floração também tende a ocorrer durante o período com menor precipitação (Janzen 1967; Daubenmire 1972; Frankie et al. 1974) ou, mais freqüentemente, durante os primeiros meses da estação chuvosa (Monasterio \& Sarmiento 1976; Morellato et al. 1989; Stranguetti \& Ranga 1997), enquanto que a frutificação pode ocorrer durante a segunda metade da estação seca (Frankie et al. 1974; Monasterio \& Sarmiento 1976; Chapman et al. 1999) ou durante a estação chuvosa (White 1994; Funch et al. 2002). Como a sazonalidade climática exerce forte influência nos padrões fenológicos anuais nas florestas sazonais, é esperado que a época de ocorrência de uma fenofase possa ser fortemente influenciada por variações interanuais na precipitação ou temperatura, as quais representem anomalias muito acima ou abaixo da média climática. Para testar estes possíveis efeitos são necessárias séries temporais de vários anos de coleta fenológica e variáveis ambientais do mesmo período do estudo (Rathcke \& Lacey 1985; Fenner 1998; Sakai 2001).

Neste estudo analisamos a queda de folhas, floração e frutificação e suas relações com três variáveis ambientais (comprimento do dia, precipitação e temperatura), durante um período de quatro anos e buscamos entender se os padrões sazonais geralmente observados em florestas estacionais semideciduais se repetem em anos consecutivos. Desta forma, buscamos responder às seguintes questões: i) Os efeitos das variações ambientais sobre o número de espécies em cada fenofase se repetem em anos consecutivos? ii) Qual variável ambiental é mais importante em cada ano para a determinação do padrão fenológico para cada fenofase? iii) Há correlação da sazonalidade fenológica entre anos sucessivos?

\section{Material e métodos}

Área de estudo - Esse estudo foi realizado em um fragmento de floresta de 240 ha de tamanho chamado Mata São José (MSJ), pertencente à fazenda São José, localizado no município de Rio Claro, estado de São Paulo, Brasil ( $22^{\circ} 25^{\prime}$ S e $47^{\circ} 28^{\prime}$ W; $630 \mathrm{~m}$ de altitude). Sua vegetação pode ser definida como floresta estacional semidecidual ou floresta semidecídua, pertencente ao domínio da Floresta Atlântica (Oliveira \& Fontes 2000). As famílias mais representativas em levantamento fitossociológico arbóreo realizado nas mesmas trilhas do presente estudo foram Euphorbiaceae, Fabaceae, Meliaceae, Myrtaceae, Rubiaceae e Rutaceae, que somaram 201 espécies de plantas com diâmetro a altura do peito (DAP) $\geq 10 \mathrm{~cm}$ (Pagano \& Leitão-Filho 1987). O dossel da MSJ pode variar entre 20 e $30 \mathrm{~m}$ de altura. Próximo às bordas do fragmento, não é possível distinguir estratificação nítida entre dossel e sub-dossel devido à presença de lianas que formam emaranhados densos e de clareiras de diferentes dimensões.

Coleta dos dados fenológicos - Ao longo de $4.500 \mathrm{~m}$ de trilhas previamente existentes em áreas de borda e interior da floresta, selecionamos e marcamos com placas de alumínio numeradas todos os indivíduos arbóreos com o diâmetro à altura do peito $\geq 10 \mathrm{~cm}$ e copa totalmente visível. Todos os indivíduos foram marcados com placas de alumínio para facilitar sua identificação. Durante quatro anos consecutivos (janeiro de 2001 a dezembro de
2004) realizamos, em intervalos mensais, observações fenológicas de 332 indivíduos arbóreos pertencentes a 90 espécies distribuídas em 37 famílias. Essa amostra representou $44,7 \%$ do total de espécies amostradas no estudo fitossociológico realizado por Pagano \& Leitão-Filho (1987). Coletamos dados sobre presença e ausência das fenofases: queda foliar, floração (flor aberta), e frutificação (frutos maduros), seguindo as definições de Morellato et al. (1989). Folhas, flores e frutos caídos sob a copa da árvore foram utilizados como indicadores da atividade fenológica em cada indivíduo. A distribuição dos indivíduos é similar à encontrada em florestas tropicais, onde muitas espécies estão representadas por poucos indivíduos e poucas espécies com muitos indivíduos. Dessa forma, em nossa amostragem, $31 \%$ das espécies possuíram apenas um indivíduo amostrado e $31 \%$ das espécies com mais de cinco indivíduos compreenderam $62 \%$ do total de indivíduos amostrados (ver Apêndice 1 para detalhes). Fournier \& Charpantier (1975) sugeriram que entre cinco a dez indivíduos representam um bom tamanho amostral para cada espécie em estudos fenológicos. No entanto, em florestas tropicais onde há alta diversidade florística e baixa densidade de espécies arbóreas, um único indivíduo amostrado de uma determinada espécie pode fornecer informações importantes sobre o comportamento fenológico da comunidade como um todo. Para discussões adicionais veja Morellato et al. (2010). A classificação botânica seguiu “Angiosperm Phylogeny Group” (APG II 2003). Sazonalidade climática da região - Na região de Rio Claro, o comprimento do dia varia entre 11 e $13 \mathrm{~h}$ ao longo do ano (Fig. 1A), onde dias mais curtos ocorrem no mesmo período das temperaturas mais baixas (Fig. 1B) e menor pluviosidade (Fig. 1C). A precipitação média anual é $1.631 \mathrm{~mm}$ e a temperatura média é $21,1^{\circ} \mathrm{C}$, considerando dados meteorológicos de 30 $\operatorname{anos}(1973$ a 2002). Durante o ano, ocorre uma estação seca e fria entre abril e setembro e uma estação chuvosa e quente de outubro a março. A estação seca e fria (inverno) geralmente possui precipitação mensal inferior a 60 mm e a estação chuvosa e quente (verão) superior a $100 \mathrm{~mm}$.

Ao longo do período de estudo (2001 a 2004), a precipitação média anual foi inferior à média do período de 30 anos, com $1.186 \mathrm{~mm}$ em 2001, 1.534 $\mathrm{mm}$ em 2002, $1.336 \mathrm{~mm}$ em 2003 e $1.472 \mathrm{~mm}$ em 2004. A temperatura média foi superior, variando entre 23 a $24^{\circ} \mathrm{C}$ nos quatro anos de monitoramento. $\mathrm{O}$ ano de 2004 foi um ano atípico em termos de temperatura, quando não houve variação marcada ao longo do ano (Fig. 1A). Todos os dados climáticos foram cedidos pelo Centro de Análise e Planejamento Ambiental (Ceapla) da Universidade Estadual Paulista (Unesp) localizada à cerca de $20 \mathrm{~km}$ da MSJ. Os dados de comprimento do dia são referentes à latitude $22^{\circ} \mathrm{S}$ e seguem Ometto (1981).
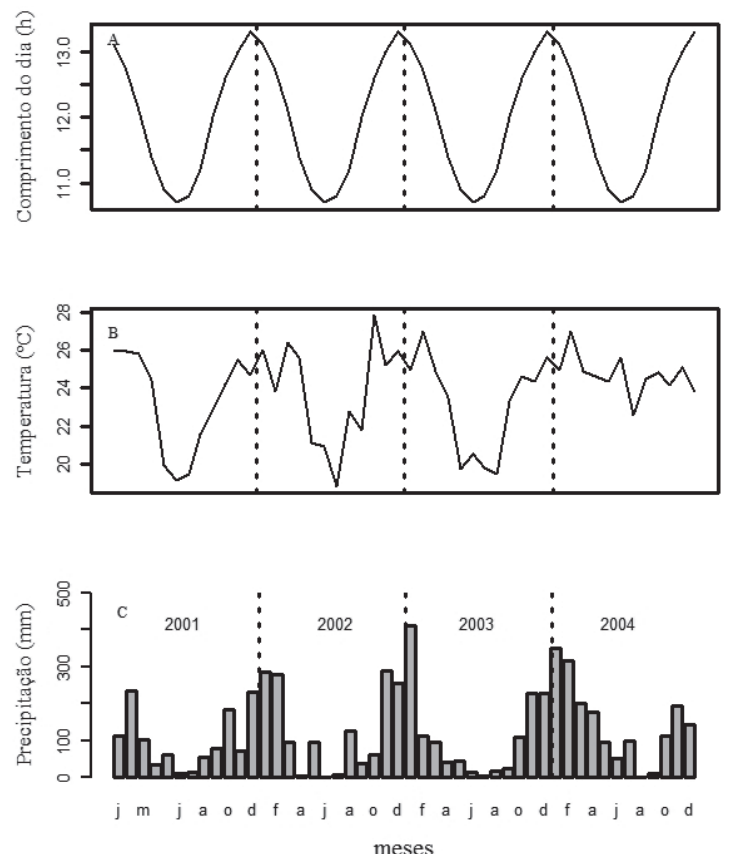

Figura 1. Variação do comprimento do dia (A), temperatura (B) e precipitação (C) durante o período de estudo (2001 a 2004) para a região de Rio Claro, SP, Brasil. 
Análise dos dados - Utilizamos regressões lineares simples para testar o efeito de cada variável climática sobre o número de espécies em cada uma das três fenofases separadamente. Para tal, ordenamos os meses de cada ano utilizando o somatório do número de espécies em uma determinada fenofase em cada mês, pareado com o valor da variável climática do mesmo mês de coleta fenológica [comprimento do dia (média mensal), precipitação (total mensal) e temperatura (média mensal)]. Após as análises de regressão, separamos os três pares de combinações formadas entre cada variável dependente (fenofase) e cada uma das três variáveis ambientais independentes em cada ano para o uso do teste de escolha de modelos, segundo o Critério de Seleção de Akaike (AIC). O AIC seleciona os melhores modelos lineares com relação ao efeito da variável preditora sobre uma determinada variável dependente, em que o modelo com menor valor de AIC é considerado como o mais explicativo (Burnham \& Anderson 2002). Utilizamos este método devido à alta correlação apresentada entre variáveis ambientais que levariam à multicolinearidade nos modelos regressões múltiplas. Para todas análises estatísticas, exceto as análises circulares, utilizamos o programa R (R Development Team 2008).

Para estimar a ocorrência e intensidade de sazonalidade entre cada uma das três fenofases entre os anos utilizamos estatística circular. Para tal, seguimos os seguintes procedimentos: 1: para cada ano de observação, calculamos a freqüência de ocorrência do evento fenológico para o total das espécies por mês; 2: os meses foram convertidos em ângulos, sendo $0^{\circ}=$ janeiro, sucessivamente até $330^{\circ}=$ dezembro, em intervalos de $30^{\circ}$; 3: calculamos o ângulo médio, o desvio padrão angular e comprimento do vetor $r$ e testamos a significância do ângulo através do teste de Rayleigh (z) para a distribuição circular (Zar 1999). As fenofases que apresentaram ângulo médio significativo $(\mathrm{p}<0,05)$ foram convertidas em data média, ou seja, data de maior intensidade da fenofase durante o ano. Para os cálculos da estatística circular foi utilizado o programa Oriana (Kovach 2004). Para testar se os eventos fenológicos significativamente sazonais que ocorreram num dado período e intensidade em cada ano se repetem entre os anos subseqüentes, aplicamos o teste a posteriori de Watson-Williams $(F)$ (Zar 1999; Morellato et al. 2000). Para verificar se as atividades fenológicas seguem o mesmo padrão em anos sucessivos, utilizamos o coeficiente de Spearman $(r)$ para correlacionar o número de espécies em determinada fenofase em um ano com o número de espécies na mesma fenofase no ano subseqüente, considerando a mesma seqüência entre os meses.

\section{Resultados}

Efeitos das variáveis climáticas - Houve um forte efeito significativo das três variáveis ambientais (comprimento do dia, precipitação e temperatura) sobre o número de es- pécies perdendo folhas para os quatro anos de estudo, com exceção da temperatura no ano de 2004 (Tab. 1). Conforme aumentaram o comprimento do dia, a pluviosidade e a temperatura, o número de espécies perdendo folhas diminuiu e vice-versa (Figs. 2A, B, C). Com base no critério de seleção de AIC, o comprimento do dia e a temperatura se alternaram como melhores preditores do número de espécies perdendo folhas (Tab. 2). Houve relação positiva significativa entre o comprimento do dia e o número de espécies florescendo para os quatro anos (Tab. 1 e Fig. 2D). A precipitação apre-
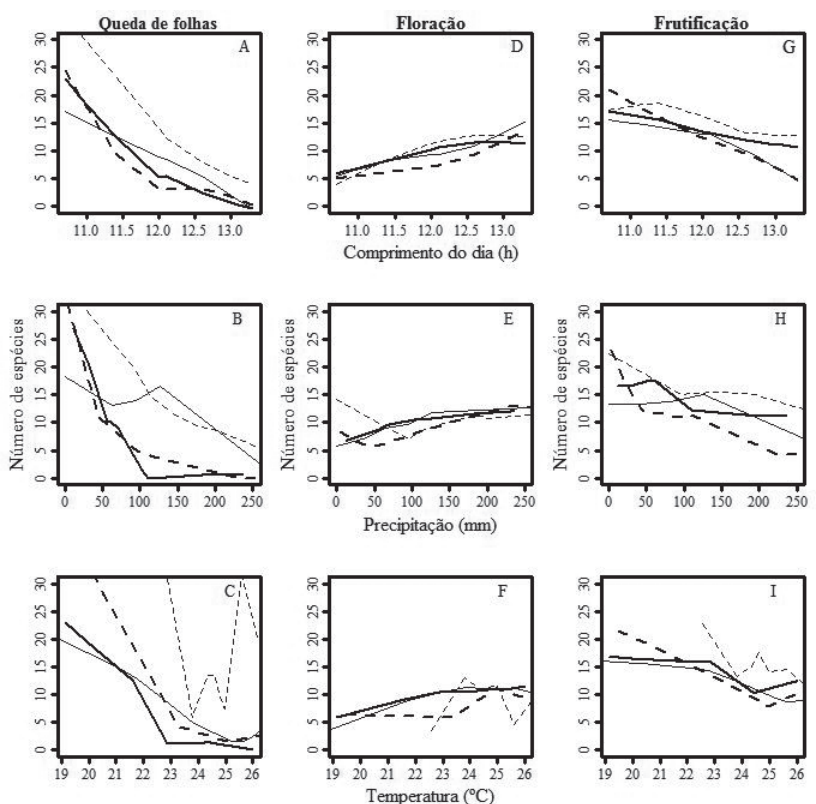

Figura 2. Tendências positivas e negativas nos números de espécies em cada fenofase em relação à variação das unidades das variáveis independentes na Mata São José, Rio Claro, SP, Brasil. A linha de tendência é composta por algoritmos para ajuste local que compõem a função lowess do programa R. Linha contínua grossa $=$ ano de 2001 , linhas contínua fina $=2002$, linha tracejada grossa $=2003$ e linha tracejada fina $=2004$.

Tabela 1. Resultado das regressões lineares relacionando três variáveis climáticas com três fenofases, para cada ano de monitoramento, em uma comunidade arbórea de floresta semidecídua em Rio Claro, SP, Brasil. $n s$ = diferença não significativa $(\mathrm{p}>0,05)$.

\begin{tabular}{|c|c|c|c|c|c|c|c|c|c|c|}
\hline & \multicolumn{4}{|c|}{ Comprimento do dia } & \multicolumn{3}{|c|}{ Precipitação } & \multicolumn{3}{|c|}{ Temperatura } \\
\hline & & $\mathrm{t}$ & $p$ & $\mathrm{R} 2$ & $\mathrm{t}$ & $p$ & R2 & $\mathrm{t}$ & $p$ & R2 \\
\hline \multirow[t]{4}{*}{ Queda de folhas } & 2001 & 0,003 & 0,005 & 0,552 & 0,003 & 0,038 & 0,362 & 0,001 & 0,001 & 0,662 \\
\hline & 2002 & 0,003 & 0,007 & 0,531 & 0,001 & 0,019 & 0,436 & 0,003 & 0,009 & 0,51 \\
\hline & 2003 & 0,016 & 0,025 & 0,407 & 0,001 & 0,025 & 0,405 & 0,003 & 0,007 & 0,524 \\
\hline & 2004 & 0,001 & 0,001 & 0,674 & 0,001 & 0,003 & 0,598 & 0,121 & $n s$ & 0,188 \\
\hline \multirow[t]{4}{*}{ Floração } & 2001 & 0,112 & 0,017 & 0,447 & 0,001 & 0,039 & 0,357 & 0,001 & 0,039 & 0,358 \\
\hline & 2002 & 0,062 & 0,016 & 0,45 & 0,001 & 0,023 & 0,416 & 0,59 & $n s$ & 0,174 \\
\hline & 2003 & 0,001 & 0,001 & 0,655 & 0,001 & 0,009 & 0,502 & 0,32 & $n s$ & 0,284 \\
\hline & 2004 & 0,085 & 0,021 & 0,423 & 0,001 & $n s$ & 0,006 & 0,377 & $n s$ & 0,139 \\
\hline \multirow[t]{4}{*}{ Frutificação } & 2001 & 0,001 & 0,01 & 0,499 & 0,001 & $n s$ & 0,235 & 0,001 & 0,003 & 0,59 \\
\hline & 2002 & 0,001 & 0,016 & 0,45 & 0,001 & 0,023 & 0,416 & 0,001 & 0,01 & 0,49 \\
\hline & 2003 & 0,007 & 0,02 & 0,429 & 0,001 & 0,033 & 0,376 & 0,017 & $n s$ & 0,307 \\
\hline & 2004 & 0,038 & $n s$ & 0,194 & 0,001 & 0,013 & 0,472 & 0,044 & $n s$ & 0,252 \\
\hline
\end{tabular}


Tabela 2. Valores estimados do Critério de Seleção de Akaike (AIC) para cada combinação de fenofase e variável ambiental em cada ano. Valores seguidos de "+" indicam à variável climática que melhor explicou a variação no número de espécies com a fenofase em um determinado ano. comp.dia = comprimento do dia; ppt = precipitação; temp = temperatura.

\begin{tabular}{|c|c|c|c|c|c|}
\hline & & \multicolumn{4}{|c|}{ AIC } \\
\hline & & 2001 & 2002 & 2003 & 2004 \\
\hline \multirow[t]{3}{*}{ Queda de folhas $\mathrm{x}$} & comp.dia & 91,956 & $80,927+$ & 99,623 & $89,132+$ \\
\hline & ppt & 96,188 & 83,133 & 99,666 & 91,662 \\
\hline & temp & $88,546+$ & 81,457 & $96,810+$ & 100,111 \\
\hline \multirow[t]{3}{*}{ Floração x } & comp,dia & $58,010+$ & $67,428+$ & $57,061+$ & $68,068+$ \\
\hline & ppt & 59,811 & 68,144 & 61,465 & 74,599 \\
\hline & temp & 59,703 & 72,308 & 65,828 & 72,882 \\
\hline \multirow[t]{3}{*}{ Frutificação x } & comp,dia & 60,787 & $64,218+$ & $77,519+$ & 76,640 \\
\hline & ppt & 65,866 & 69,041 & 78,589 & $71,572+$ \\
\hline & temp & $58,387+$ & 67,596 & 79,848 & 75,744 \\
\hline
\end{tabular}

sentou um efeito positivo significativo sobre o número de espécies florescendo para os três primeiros anos (2001 a 2003) e temperatura somente no ano de 2001 (Tab. 1). Para todos os anos, o comprimento do dia foi a melhor variável para predizer o número de espécies florescendo (Tab. 2). O número de espécies em frutificação apresentou relação significativa com o comprimento do dia nos três primeiros anos, com a precipitação em 2002 e 2003 e com a temperatura em 2001 e 2002 (Tab. 1). Os gráficos com linhas de tendência apresentam um maior número de espécies em frutificação conforme se diminui o comprimento do dia, precipitação e temperatura (Fig. 2G-I). Os melhores modelos segundo o AIC se alternaram entre as três variáveis climáticas nos quatro anos (Tab. 2).

Sazonalidade das três fenofases e relação entre anos Não houve correlação significativa entre os anos de 2003 e 2004 para o número de espécies florescendo e frutificando, ao passo que o número de espécies perdendo folhas foi correlacionado para todos os anos sucessivos (Tab. 3). Os testes de estatística circular indicaram forte sazonalidade para queda foliar e data média durante o mês de julho, independentemente do ano (Tab. 4). No entanto, o teste a posteriori de Watson-Williams ( F-test $)$ evidenciou que apenas os anos de 2003 e 2004 diferiram $(F=4,603, p=0,033)$. Por outro lado, não foi detectada sazonalidade significativa na floração e frutificação da comunidade em nenhum dos anos (Tab. 4).

\section{Discussão}

O comprimento do dia, uma das variáveis que mede o fotoperíodo, varia muito pouco ao longo do ano em áreas próximas ao equador. No entanto, nas regiões acima de $15^{\circ}$ de latitude, onde a variação anual e a previsibilidade da sazonalidade climática são mais evidentes, o comprimento do dia pode ser um fator que controla a manifestação de tais eventos. Alguns estudos sugerem que pequenas mudanças no comprimento do dia ao longo do ano podem regular o comportamento fenológico das plantas, mesmo em florestas próximas ao Equador com sazonalidade climática pouco evidente (Wright \& van Schaik 1994; Morellato et al. 2000; Marques et al. 2004; Borchert et al. 2005; Zimmerman et al. 2007; Stevenson et al. 2008). Na região onde foi desenvolvido este estudo (latitude $22^{\circ} \mathrm{S}$ ), o comprimento do dia possui amplitude de cerca de duas horas ao longo do ano. Porém, apesar da evidente sazonalidade intra-anual, e ao contrário da temperatura e precipitação, o comprimento do dia não é uma variável ambiental estocástica. Assim, esta variável foi a melhor preditora para os três eventos fenológicos aqui analisados, corroborando com os estudos fenológicos realizados em outras florestas semidecíduas no sudeste brasileiro (Morellato et al. 1989; Stranguetti \& Ranga 1997), onde ocorrem um maior número de espécies em frutificação durante os dias mais curtos, como conseqüência da maioria das espécies florescer durante os dias mais longos.

Por outro lado, embora haja certa previsibilidade na variação de temperatura e precipitação em regiões tropicais de alta latitude ao longo de um mesmo ano, onde há períodos marcadamente frios e secos, podem ocorrer diferenças na quantidade e distribuição da precipitação e de temperatura entre anos. De fato, no período que abrangeu este estudo, houve uma anomalia climática em termos de temperatura em 2004, quando a temperatura nos meses mais frios (maio a agosto) foi, em média, cerca de $20 \%$ mais elevada do que a média climática da região e do que nos outros três anos de estudo. Coincidentemente, naquele ano a temperatura não afetou o número de espécies nas três fenofases. Embora o aumento da temperatura possa atuar como um estímulo à floração em espécies arbóreas tropicais (Bhat 1992; Singh \& Singh 1992; Wright \& van Schaik 1994), invernos relati- 
Tabela 3. Coeficientes de correlação de Spearman (rs) para as três fenofases entre os anos sucessivos em uma comunidade arbórea de floresta semidecídua em Rio Claro, SP, Brasil. $n s=$ diferença não significativa $(\mathrm{p}>0,05)$.

\begin{tabular}{|c|c|c|c|c|c|c|}
\hline & \multicolumn{2}{|c|}{$2001 \times 2002$} & \multicolumn{2}{|c|}{$2002 \times 2003$} & \multicolumn{2}{|c|}{$2003 \times 2004$} \\
\hline & $r_{s}$ & $p$ & $r_{s}$ & $p$ & $r_{s}$ & $p$ \\
\hline Queda de folhas & 0,869 & 0,000 & 0,861 & 0,000 & 0,783 & 0,002 \\
\hline Floração & 0,557 & $n s$ & 0,639 & 0,025 & 0,383 & $n s$ \\
\hline Frutificação & 0,813 & 0,001 & 0,657 & 0,02 & 0,42 & $n s$ \\
\hline
\end{tabular}

Tabela 4. Resultados dos testes de estatística circular para ocorrência de sazonalidade e data média para três fenofases para cada ano em uma comunidade arbórea de floresta semidecídua em Rio Claro, SP, Brasil. * = somatório anual do número de observações de cada fenofase; $n s=$ diferença não significativa ( $\mathrm{p}>0,05) ;-=$ sem data média calculada.

\begin{tabular}{|c|c|c|c|c|c|c|c|}
\hline Fenofase & Ano & $\begin{array}{c}\text { Número de } \\
\text { observações* }\end{array}$ & $\begin{array}{l}\text { Ângulo } \\
\text { médio }\end{array}$ & $\begin{array}{c}\text { Desvio } \\
\text { padrão circular }\end{array}$ & $\begin{array}{l}\text { Data } \\
\text { média }\end{array}$ & $\mathrm{r}$ & $\begin{array}{c}\text { Rayleigh } \\
(P)\end{array}$ \\
\hline \multirow[t]{4}{*}{ Queda de folhas } & 2001 & 124 & $186,94^{\circ}$ & $39,37^{\circ}$ & 6/jul & 0,79 & $<0,001$ \\
\hline & 2002 & 106 & $194,61^{\circ}$ & $54,93^{\circ}$ & 14/jul & 0,632 & $<0,001$ \\
\hline & 2003 & 165 & $196,65^{\circ}$ & $47,52^{\circ}$ & 16/jul & 0,709 & $<0,001$ \\
\hline & 2004 & 201 & $183,60^{\circ}$ & $63,20^{\circ}$ & $1 / \mathrm{jul}$ & 0,544 & $<0,001$ \\
\hline \multirow[t]{4}{*}{ Floração } & 2001 & 115 & $307,58^{\circ}$ & $110,14^{\circ}$ & - & 0,158 & $n s$ \\
\hline & 2002 & 118 & $308,22^{\circ}$ & $98,31^{\circ}$ & - & 0,229 & $n s$ \\
\hline & 2003 & 98 & $318,75^{\circ}$ & $94,63^{\circ}$ & - & 0,256 & $n s$ \\
\hline & 2004 & 127 & $307,88^{\circ}$ & $102,30^{\circ}$ & - & 0,203 & $n s$ \\
\hline \multirow[t]{4}{*}{ Frutificação } & 2001 & 163 & $173,34^{\circ}$ & $115,30^{\circ}$ & - & 0,132 & $n s$ \\
\hline & 2002 & 136 & $158,52^{\circ}$ & $99,11^{\circ}$ & - & 0,224 & $n s$ \\
\hline & 2003 & 142 & $174,21^{\circ}$ & $93,21^{\circ}$ & - & 0,266 & $n s$ \\
\hline & 2004 & 189 & $205,57^{\circ}$ & $108,13^{\circ}$ & - & 0,168 & $n s$ \\
\hline
\end{tabular}

vamente quentes implicam que as plantas poderão continuar investindo em crescimento vegetativo em vez de alterar a fase para reprodução. Em regiões tropicais onde a estação seca é severa o suficiente, tal qual a deste estudo, não investir nem em reprodução e nem em crescimento, na época de precipitação e temperaturas mais baixas, pode ser uma estratégia adaptativa das plantas em resposta à forte variação anual na disponibilidade de água do solo (Corlett \& Lafrankie 1988). Neste estudo, a precipitação exerceu um efeito positivo no número de espécies florescendo e frutificando para alguns anos. Evidências sugerem que as primeiras chuvas após a seca podem exercer forças desencadeadoras da floração em várias espécies arbóreas tropicais (Alvin \& Alvin 1976; Opler et al. 1976; Borchert et al. 2004) e assim, influenciar o padrão de floração das comunidades, por exemplo nas florestas semideciduais do sudeste brasileiro (Morellato et al. 1989; Stranguetti \& Ranga 1997) e florestas sazonais na Venezuela (Ramirez 2002), Índia (Singh \& Kushwaha 2006) e Costa do Marfim (Anderson et al. 2005). Portanto, com base nas relações causais das variáveis ambientais analisa- das, mudanças sazonais na precipitação e temperatura ao longo do ano puderam explicar em grande parte os padrões fenológicos observados neste estudo.

O padrão evidentemente sazonal e previsível e com pouca variação entre anos foi encontrado somente para a queda de folhas na Mata São José. O caráter sazonal da queda de folhas das florestas semideciduais brasileiras (Morellato et al. 1989; Stranguetti \& Ranga 1997) e das florestas tropicais com marcante sazonalidade climática no Neotrópico (Janzen 1967; Daubenmire 1972; Frankie et al. 1974; Monasterio \& Sarmiento 1976) tem sido evidenciado em diversos estudos. Entretanto, pouco se conhece sobre a variação da queda de folhas entre anos sucessivos. Os resultados deste estudo, embora com diferença estatística apenas entre os anos de 2003 e 2004, sugerem que esta fenofase é bastante previsível, pois houve correlações significativas para esta fenofase e variáveis climáticas entre os anos sucessivos. Ao contrário, os padrões de floração e frutificação não foram significativamente sazonais para todos os anos de estudo na Mata São José, diferindo daqueles descritos para outras 
florestas semideciduais do sudeste do Brasil (Morellato et al. 1989; Morellato \& Leitão Filho 1996; Stranguetti \& Ranga 1997). Além disso, para essas duas fenofases não houve padrão semelhante no número de espécies florescendo e frutificando entre os anos sucessivos, indicando que a intensidade destas duas fenofases pode estar relacionada a variações interanuais de precipitação e temperatura. De fato, o ano de 2004, quando a temperatura média foi mais elevada, não apresentou correlação significativa com o ano anterior.

Os impactos das mudanças climáticas sobre as florestas tropicais dependerão da taxa de aquecimento (aumento da temperatura) e da concomitante mudança nos regimes das chuvas em regiões tipicamente sazonais (Wright \& MullerLandau 2006). É esperado que em regiões tropicais de alta latitude ocorra aumento da temperatura e diminuição do período seco ocasionado por fenômenos climáticos extremos, tais como o El-Niño, que vêm se tornando cada vez mais frequentes (Marengo et al. 2009). Asthon et al. (1988) mostraram que a variabilidade climática ocasionada pelo El-Niño em um determinado ano teve um forte efeito na fenologia reprodutiva em florestas não sazonais na Ásia, com possíveis impactos no sucesso reprodutivo das plantas. $\mathrm{O}$ ano de 2004, considerado atípico em termos de temperatura, não mostrou um efeito significativo desta variável no número de espécies florescendo e frutificando e, além disso, este ano não foi correlacionado com o ano anterior para estas duas fenofases. Séries mais longas de registros fenológicos poderão fornecer maior confiança sobre as relações entre as variações interanuais nos padrões fenológicos com a temperatura e precipitação e, portanto, permitir melhor inferência sobre os efeitos das mudanças climáticas nos trópicos. Além disso, os efeitos diretos das mudanças climáticas podem ser menos sérios do que os efeitos indiretos, por exemplo, através do impacto das mudanças nos padrões fenológicos sobre os agentes polinizadores e dispersores de sementes (Kelly \& Sork 2002).

\section{Agradecimentos}

Os autores agradecem aos proprietários da Fazenda São José por permitir o desenvolvimento da pesquisa na área; à FAPESP pelas bolsas de Iniciação Científica (proc. 01/09575-9) e de Mestrado (proc. 03/11131-7) concedidas à PR; ao CNPq pela Bolsa de Produtividade em Pesquisa de LPCM e HEMN.

\section{Referências bibliográficas}

Alvin, P.T. \& Alvin, R. 1976. Tropical trees as living systems. Cambridge, Cambridge University Press.

Anderson, D.P.; Nordheim, E.V.; Moermond, T.C.; Bi, Z.B.G. \& Boesch, C. 2005. Factors influencing tree phenology in Tai National Park, Cote d'Ivoire. Biotropica 37: 631-640.

Angiosperm Phylogeny Group II . 2003. An update of the Angiosperm Phylogeny Group classification for the orders and families of flowering plants: APG II. Botanical Journal of the Linnean Society 141: 399-436.

Asthon, P.S; Givnish, T.J. \& Appanah, S. 1988. Staggered flowering in the Dipterocarpaceae: new insights into floral induction and the evolution of mast fruiting in the seasonal tropics. American Naturalist 132: 44-66.

Bhat, D.M. 1992. Phenology of tree species of tropical moist forest of Uttara Kannada District, Karnataka, India. Journal of Biosciences 17: $325-352$
Bolmgren, K. \& Cowan, P.D. 2008. Time-size tradeoffs: a phylogenetic comparative study of flowering time, plant height and seed mass in a north-temperate flora. Oikos 117: 424-429.

Borchert, R.; Rivera, G. \& Hagnauer, W. 2002. Modification of vegetative phenology in a tropical semi-deciduous forest by abnormal drought and rain. Biotropica 34: 27-39.

Borchert, R.; Meyer, S.A.; Felger, R.S. \& Porter-Bolland, L. 2004. Environmental control of flowering periodicity in Costa Rican and Mexican tropical dry forests. Global Ecology and Biogeography 13: 409-425.

Borchert, R.; Renner, S.S.; Calle, Z.; Navarrete, D.; Tye, A.; Gautier, L.; Spichiger, R. \& Von Hildebrand, P. 2005. Photoperiodic induction of synchronous flowering near the Equator. Nature 433: 627-629.

Boulter, S.L.; Kitching, R.L. \& Howlett, B.G. 2006. Family, visitors and the weather: patterns of flowering in tropical rain forests of northern Australia. Journal of Ecology 94: 369-82.

Burnham, K.P. \& Anderson, D.R. 2002. Model selection and multimodel inference: a practical information-theoretic approach. 2nd. New York, Springer-Verlag.

Chapman, C.A.; Wrangham, R.W.; Chapman, L.J.; Kennard, D.K. \& Zanne, A.E. 1999. Fruit and flower phenology at two sites in Kibale National Park, Uganda. Journal of Tropical Ecology 15: 189-211.

Corlett, R. \& Lafrankie, J.V. 1988. Potential impacts of climate change on tropical forests through an influence on phenology. Climatic Change 39: 439-453.

Daubenmire, R. 1972. Phenology and other characteristics of tropical semideciduous forest in north-western Costa Rica. Journal of Ecology 60: $147-170$

Fenner, M. 1998. The phenology of growth and reproduction in plants. Perspectives in Plant Ecology, Evolution and Systematics 1: 78-91

Fournier, L.A. \& Charpantier, C. 1975. El tamaño de la muestra y la frecuencia de las observaciones en el estudio de las características fenológicas de los árboles tropicales. Turrialba 25: 45-48.

Frankie, G.W.; Baker, H.G. \& Opler, P.A. 1974. Comparative phenological studies of trees in tropical wet $\&$ dry forest in the lowlands of Costa Rica. Journal of Ecology 62: 881-919.

Funch, L.S.; Funch, R. \& Barroso, G.M. 2002. Phenology of gallery and montane forest in the Chapada Diamantina, Bahia, Brazil. Biotropica 34: $40-50$.

Janzen, D.H. 1967. Synchronization of sexual reproduction of trees within dry season in Central America. Evolution 21: 620-637.

Kelly, D. \& Sork, V.L. 2002. Mast seeding in perennial plants?: Why, how, where? Annual Review of Ecology and Systematics 33: 427-447.

Kovach. 2004. Oriana for Windows. Wales, Kovach Computing Services.

Laurance, W.F.; Oliveira, A.A.; Laurance, S.G.; Condit, R.; Nascimento, H.E.M.; Sanchez-Thorin, A.C.; Lovejoy, T.E.; Andrade, A.; D’Angelo, S.; Ribeiro, J.E. \& Dick, C.W. 2004. Pervasive alteration of tree communities in undisturbed Amazonian forests. Nature 428: 171-175.

Leigh, E.G. \& Windsor, D.M. 1982. Forest production regulation of primary consumers on Barro Colorado Island. Washington, D.C. Smithsonian Institution Press.

Marengo, J.A.; Jones, R.; Alves, L.M. \& Valverde, M.C. 2009. Future change of temperature and precipitation extremes in South America as derived from the PRECIS regional climate modeling system. International Journal of Climatology 29: 2241-2255.

Marques, M.C.M.; Roper, J.J. \& Salvalaggio, A.P.B. 2004. Phenological patterns among plant life-forms in a subtropical forest in southern Brazil. Plant Ecology 173: 203-213.

Menzel, A.; Sparks, T.H.; Estrella, N.; Koch, E.; Aasa, A.; Ahas, R.; AlmKubler, K.; Bissolli, P.; Braslavska, O.; Briede, A.; Chmielewski, F.M.; Crepinsek, Z.; Curnel, Y.; Dahl, A.; Defila, C.; Donnelly, A.; Filella, Y.; Jatcza, K.; Mage, F.; Mestre, A.; Nordli, O.; Penuelas, J.; Pirinen, P.; Remisova, V.; Scheifinger, H.; Striz, M.; Susnik, A.; Van Vliet, A.J.H.; Wielgolaski, F.E.; Zach, S. \& Zust, A. 2006. European phenological response to climate change matches the warming pattern. Global Change Biology 12: 1969-76.

Monasterio, M. \& Sarmiento, G. 1976. Phenological strategies of plants species in the tropical savanna \& semi-deciduous forest of the Venezuelan Llanos. Journal of Biogeography 3: 325-356.

Morellato, L.P.C. \& Leitão-Filho, H.F. 1996. Reproductive phenology of climbers in a southeastern Brazilian forest. Biotropica 28: 180-191. 
Morellato, L.P.C.; Rodrigues, R.R.; Leitão-Filho, H.F. \& Joly, C.A. 1989. Estudo comparativo da fenologia de espécies arbóreas em floresta de altitude e floresta mesófila semidecídua na Serra do Japi, Jundiaí, São Paulo. Revista Brasileira de Botânica 12: 85-98.

Morellato, L.P.C.; Talora, D.C.; Takahasi, A.; Bencke, C.C.; Romera, E.C. \& Zipparro, V.B. 2000. Phenology of Atlantic rain forest trees: A comparative study. Biotropica 32: 811-823.

Morellato, L.P.C.; Alberti, L.F. \& Hudson, I.L. 2010 Applications of circular statistics in plant phenology: a case studies approach. Pp. 357371. In: Keatley, M. \& Hudson, I.L. (eds.) Phenological Research: Methods for Environmental and Climate Change Analysis. New York, Springer.

Oliveira, A.T. \& Fontes, M.A.L. 2000. Patterns of floristic differentiation among Atlantic forests in southeastern Brazil and the influence of climate. Biotropica 32: 793-810.

Ometto, J.C. 1981, Bioclimatologia vegetal. Piracicaba, Agronomica Ceres.

Opler, P.A.; Frankie, G.W. \& Baker, H.G. 1976. Rainfall as a factor in the release, timing and synchronization of anthesis by tropical trees and shrubs. Journal of Biogeography 3: 231-236.

Pagano, S.N. \& Leitão-Filho, H.F. 1987. Estudo florístico do estrato arbóreo de mata mesófila semidecídua, no município de Rio Claro (Estado de São Paulo). Revista Brasileira de Botânica 10: 37-47.

Phillips, O.L.; Baker, T.R.; Arroyo, L.; Higuchi, N.; Killeen, T.J.; Laurance, W.F.; Lewis, S.L.; Lloyd, J.; Malhi, Y.; Monteagudo, A.; Neill, D.A.; Vargas, P.N.; Silva, J.N.M.; Terborgh, J.; Martinez, R.V.; Alexiades, M.; Almeida, S.; Brown, S.; Chave, J. Comiskey, J.A.; Czimczik, C.I.; Di Fiore, A.; Erwin, T.; Kuebler, C.; Laurance, S.G.; Nascimento, H.E.M.; Olivier, J.; Palacios, W.; Patino, S.; Pitman, N.C.A.; Quesada, C.A.; Salidas, M.; Lezama, A.T. \& Vinceti, B. 2004. Pattern and process in Amazon tree turnover, 1976-2001. Philosophical Transactions of the Royal Society of London Series B-Biological Sciences 359: 381-407.

Post E.S.; Pedersen C.; Wilmers, C.C. \& Forchhammer, M.C. 2008. Phenological sequences reveal aggregate life history response to climatic warming. Ecology 89: 363-70.

R Development Team. 2008. A language and environment for statistical computing. Vienna, R Foundation for Statistical Computing.

Ramírez, N. 2002. Reproductive phenology, life-forms, and habitats ofthe Venezuelan Central Plain. American Journal of Botany 89: 836-842.
Rathcke, B. \& Lacey, E.P. 1985. Phenological patterns of terrestrial plants. Annual Review of Ecology and Systematics 16: 179-214.

Reich, P.B. 1995. Phenology of tropical forests - patterns, causes, and consequences. Canadian Journal of Botany 73: 164-174.

Reich, P.B. \& Borchert, R. 1984. Water-stress and tree phenology in a Tropical Dry Forest in the lowlands of Costa-Rica. Journal of Ecology 72: 61-74.

Sakai, S. 2001. Phenological diversity in tropical forests. Population Ecology 43: 77-86

Singh, J.S. \& Singh, V.K. 1992. Phenology of a seasonally dry tropical forest. Current Science 63: 684-689.

Singh, K.P. \& Kushwaha, C.P. 2006. Diversity of flowering and fruiting phenology of trees in a tropical deciduous forest in India. Annals of Botany 97: 265-276.

Stevenson, P.R.; Castellanos, M.C.; Cortés, A.I. \& Link, A. 2008. Flowering patterns in a seasonal tropical lowland forest in western Amazonia. Biotropica 40: 559-567.

Stranguetti, V. \& Ranga, N.T. 1997. Phenological aspects of flowering \& fruiting at the Ecological Station of Paulo de Faria-SP-Brazil. Tropical Ecology 38: 323-327.

van Schaik, C.P.; Terborgh, J.W. \& Wright, S.J. 1993. The phenology of tropical forest: adaptative significance and consequences of consumers. Annual Review of Ecology and Systematics 24: 353-377.

White, L.J.T. 1994. Patterns of fruit-fall phenology in the Lope-Reserve, Gabon. Journal of Tropical Ecology 10: 289-312.

Wright, S.J. \& van Schaik, C.P. 1994. Light and the phenology of tropical trees. American Naturalist 143: 192-199.

Wright, S.J. \& Calderon, O. 2006. Seasonal, El Niño and longer term changes in flower and seed production in a moist tropical forest. Ecology Letters 9: 35-44.

Wright, S.J. \& Muller-Landau, H.C. 2006. The future of tropical forest species. Biotropica 38: 287-301.

Zar, J.H. 1999. Bioestatistical Analysis. New Jersey, Prentice Hall.

Zimmerman, J.K.; Wright, S.J.; Calderón, O.; Pagan, M.A. \& Paton, S. 2007. Flowering and fruiting phenologies of seasonal and aseasonal neotropical forests: the role of annual changes in irradiance. Journal of Tropical Ecology 23: 231-251. 
Apêndice 1. Lista das espécies estudadas na Mata São José, Rio Claro, SP, Brasil, com indicação da atividade de cada fenofase (x) nos anos registrados. N = número de indivíduos.

\begin{tabular}{|c|c|c|c|c|c|c|c|c|c|c|c|c|c|c|}
\hline \multirow[t]{2}{*}{ Família } & \multirow[t]{2}{*}{ Espécie } & \multirow[t]{2}{*}{$\mathrm{N}$} & \multicolumn{4}{|c|}{ Queda foliar } & \multicolumn{4}{|c|}{ Floração } & \multicolumn{4}{|c|}{ Frutificação } \\
\hline & & & 2001 & 2002 & 2003 & 2004 & 2001 & 2002 & 2003 & 2004 & 2001 & 2002 & 2003 & 2004 \\
\hline Anacardiaceae & Astronium graveolens Jacq. & 10 & $\mathrm{x}$ & $\mathrm{x}$ & $\mathrm{x}$ & $\mathrm{x}$ & $\mathrm{x}$ & $\mathrm{x}$ & & & $\mathrm{x}$ & & & \\
\hline \multirow[t]{3}{*}{ Annonaceae } & Annona cacans Warm. & 5 & $\mathrm{x}$ & $\mathrm{x}$ & $\mathrm{x}$ & $\mathrm{x}$ & $\mathrm{x}$ & $\mathrm{x}$ & $\mathrm{x}$ & $\mathrm{x}$ & $\mathrm{x}$ & $\mathrm{x}$ & $\mathrm{x}$ & $\mathrm{x}$ \\
\hline & Duguetia lanceolata A. St.-Hil. & 1 & & & & & $\mathrm{x}$ & $\mathrm{x}$ & & $\mathrm{x}$ & $\mathrm{x}$ & & & \\
\hline & Xylopia brasiliensis Spreng. & 2 & & & & & & & & & $\mathrm{x}$ & & & \\
\hline Apocynaceae & Aspidosperma polyneuron Müll. Arg. & 8 & $\mathrm{x}$ & $\mathrm{x}$ & $\mathrm{x}$ & $\mathrm{x}$ & & $\mathrm{x}$ & $\mathrm{x}$ & $\mathrm{x}$ & $\mathrm{x}$ & $\mathrm{x}$ & $\mathrm{x}$ & $\mathrm{x}$ \\
\hline \multirow[t]{3}{*}{ Araliaceae } & Aralia excelsa (Griseb.) I. Wen & 1 & & $\mathrm{x}$ & $\mathrm{x}$ & & $\mathrm{x}$ & & & $\mathrm{x}$ & & $\mathrm{x}$ & & \\
\hline & Dendropanax cuneatus (DC.) Decne. \& Planch. & 2 & & & & $\mathrm{x}$ & & $\mathrm{x}$ & & & & & & \\
\hline & Schefflera morototoni (Aubl.) Maquire, Steyem. \& Frodin & 1 & & & & & $\mathrm{x}$ & $\mathrm{x}$ & $\mathrm{x}$ & $\mathrm{x}$ & & & & $\mathrm{x}$ \\
\hline \multirow[t]{3}{*}{ Arecaceae } & Euterpe edulis Mart. & 6 & & & & & $\mathrm{x}$ & $\mathrm{x}$ & $\mathrm{x}$ & $\mathrm{x}$ & $\mathrm{x}$ & $\mathrm{x}$ & $\mathrm{x}$ & $\mathrm{x}$ \\
\hline & Syagrus oleracea (Mart.) Becc. & 4 & & & & & $\mathrm{x}$ & $\mathrm{x}$ & $\mathrm{x}$ & $\mathrm{x}$ & $\mathrm{x}$ & $\mathrm{x}$ & $\mathrm{x}$ & $\mathrm{x}$ \\
\hline & Syagrus romanzoffiana (Cham.) Glassman & 11 & & & & & $\mathrm{x}$ & $\mathrm{x}$ & $\mathrm{x}$ & $\mathrm{x}$ & $\mathrm{x}$ & $\mathrm{x}$ & $\mathrm{x}$ & $\mathrm{x}$ \\
\hline \multirow[t]{2}{*}{ Asteraceae } & Piptocarpha rotundifolia (Less.) Baker & 2 & $\mathrm{x}$ & & & & $\mathrm{x}$ & & $\mathrm{x}$ & $\mathrm{x}$ & $\mathrm{x}$ & $\mathrm{x}$ & & \\
\hline & Vernonia diffusa Less. & 6 & $\mathrm{x}$ & $\mathrm{x}$ & $\mathrm{x}$ & $\mathrm{x}$ & $\mathrm{x}$ & $\mathrm{x}$ & $\mathrm{x}$ & $\mathrm{x}$ & $\mathrm{x}$ & $\mathrm{x}$ & $\mathrm{x}$ & \\
\hline \multirow[t]{2}{*}{ Bignoniaceae } & Jacaranda macrantha Cham. & 2 & $\mathrm{x}$ & $\mathrm{x}$ & $\mathrm{x}$ & $\mathrm{x}$ & & $\mathrm{x}$ & & $\mathrm{x}$ & & & & \\
\hline & Zeyhera tuberculosa (Vell.) Bureau & 1 & & & $\mathrm{x}$ & $\mathrm{x}$ & & & & & & & & \\
\hline Boraginaceae & Cordia ecalyculata Vell. & 1 & & & & & & $\mathrm{x}$ & $\mathrm{x}$ & & $\mathrm{x}$ & & $\mathrm{x}$ & \\
\hline Burseraceae & Protium heptaphyllum (Aubl.) Marchand & 1 & & $\mathrm{x}$ & & $\mathrm{x}$ & & & $\mathrm{x}$ & & $\mathrm{x}$ & $\mathrm{x}$ & $\mathrm{x}$ & $\mathrm{x}$ \\
\hline Cannabaceae & Celtis iguanaea (Jacq.) Sarg. & 1 & & & & & $\mathrm{x}$ & & & & $\mathrm{x}$ & & & \\
\hline Caricaceae & Jacaratia spinosa (Aubl.) A. DC. & 1 & $\mathrm{x}$ & $\mathrm{x}$ & $\mathrm{x}$ & $\mathrm{x}$ & & & $\mathrm{x}$ & & $\mathrm{x}$ & $\mathrm{x}$ & $\mathrm{x}$ & $\mathrm{x}$ \\
\hline Celastraceae & Maytenus aquifolium Mart. & 1 & & & & & & & & & & & & \\
\hline Clusiaceae & Calophyllum brasiliense Cambess. & 11 & & & & & & & $\mathrm{x}$ & & $\mathrm{x}$ & $\mathrm{x}$ & $\mathrm{x}$ & $\mathrm{x}$ \\
\hline Combretaceae & Terminalia brasiliensis (Cambess. ex A. St.-Hil.) Eichler & 1 & $\mathrm{x}$ & & $\mathrm{x}$ & & & $\mathrm{x}$ & & & & $\mathrm{x}$ & $\mathrm{x}$ & \\
\hline \multirow[t]{9}{*}{ Euphorbiacae } & Actinostemon estrellensis (Müll. Arg.) Pax & 3 & & & & $\mathrm{x}$ & $\mathrm{x}$ & & $\mathrm{x}$ & & & & & $\mathrm{x}$ \\
\hline & Croton floribundus Spreng. & 11 & $\mathrm{x}$ & $\mathrm{x}$ & $\mathrm{x}$ & $\mathrm{x}$ & $\mathrm{x}$ & $\mathrm{x}$ & $\mathrm{x}$ & $\mathrm{x}$ & $\mathrm{x}$ & & $\mathrm{x}$ & $\mathrm{x}$ \\
\hline & Croton piptocalyx Müll. Arg. & 6 & $\mathrm{x}$ & $\mathrm{x}$ & $\mathrm{x}$ & $\mathrm{x}$ & $\mathrm{x}$ & $\mathrm{x}$ & $\mathrm{x}$ & $\mathrm{x}$ & $\mathrm{x}$ & $\mathrm{x}$ & $\mathrm{x}$ & $\mathrm{x}$ \\
\hline & Croton salutaris Casar. & 1 & $\mathrm{x}$ & & $\mathrm{x}$ & $\mathrm{x}$ & & $\mathrm{x}$ & & & & & & \\
\hline & Sapium glandulatum (Vell.) Plax & 2 & $\mathrm{x}$ & $\mathrm{x}$ & $\mathrm{x}$ & $\mathrm{x}$ & & & & $\mathrm{x}$ & & & & $\mathrm{x}$ \\
\hline & Savia dictyocarpa Müll. Arg. & 6 & $\mathrm{x}$ & $\mathrm{x}$ & $\mathrm{x}$ & $\mathrm{x}$ & $\mathrm{x}$ & $\mathrm{x}$ & $\mathrm{x}$ & & $\mathrm{x}$ & $\mathrm{x}$ & $\mathrm{x}$ & $\mathrm{x}$ \\
\hline & Sebastiania brasiliensis Spreng. & 1 & & & $\mathrm{x}$ & $\mathrm{x}$ & & & & $\mathrm{x}$ & & & & \\
\hline & Sebastiania commersoniana (Baill.) L.B. Sm.\& Downs & 3 & $\mathrm{x}$ & & $\mathrm{x}$ & $\mathrm{x}$ & & $\mathrm{x}$ & & $\mathrm{x}$ & & & & \\
\hline & Sebastiania klotzschiana (Müll. Arg.) Müll. Arg. & 1 & & & $\mathrm{x}$ & & $\mathrm{x}$ & & & & & & $\mathrm{x}$ & \\
\hline \multirow[t]{12}{*}{ Fabaceae } & Bauhinia forficata Link. & 4 & $\mathrm{x}$ & $\mathrm{x}$ & $\mathrm{x}$ & $\mathrm{x}$ & $\mathrm{x}$ & $\mathrm{x}$ & $\mathrm{x}$ & $\mathrm{x}$ & $\mathrm{x}$ & $\mathrm{x}$ & $\mathrm{x}$ & $\mathrm{x}$ \\
\hline & Copaifera langsdorffii Desf. & 9 & $\mathrm{x}$ & & $\mathrm{x}$ & $\mathrm{x}$ & $\mathrm{x}$ & $\mathrm{x}$ & $\mathrm{x}$ & & & & $\mathrm{x}$ & \\
\hline & Holocalyx balansae Micheli & 4 & & $\mathrm{x}$ & & & & $\mathrm{x}$ & & & $\mathrm{x}$ & $\mathrm{x}$ & $\mathrm{x}$ & \\
\hline & Hymenaea courbaril $\mathrm{L}$. & 5 & $\mathrm{x}$ & & $\mathrm{x}$ & $\mathrm{x}$ & & & $\mathrm{x}$ & $\mathrm{x}$ & $\mathrm{x}$ & & $\mathrm{x}$ & $\mathrm{x}$ \\
\hline & Anadenanthera peregrina (L.) Speg & 11 & $\mathrm{x}$ & $\mathrm{x}$ & $\mathrm{x}$ & $\mathrm{x}$ & $\mathrm{x}$ & $\mathrm{x}$ & $\mathrm{x}$ & $\mathrm{x}$ & $\mathrm{x}$ & $\mathrm{x}$ & $\mathrm{x}$ & $\mathrm{x}$ \\
\hline & Inga sessilis (Vell.) Mart. & 4 & & & $\mathrm{x}$ & $\mathrm{x}$ & $\mathrm{x}$ & $\mathrm{x}$ & $\mathrm{x}$ & $\mathrm{x}$ & & & & \\
\hline & Inga uruguensis Hook. \& Arn. & 2 & & $\mathrm{x}$ & $\mathrm{x}$ & $\mathrm{x}$ & & $\mathrm{x}$ & $\mathrm{x}$ & $\mathrm{x}$ & & & & \\
\hline & Piptadenia gonoacantha (Mart.) J.F. Macbr. & 3 & & $\mathrm{x}$ & & $\mathrm{x}$ & $\mathrm{x}$ & $\mathrm{x}$ & $\mathrm{x}$ & & $\mathrm{x}$ & $\mathrm{x}$ & $\mathrm{x}$ & $\mathrm{x}$ \\
\hline & Centrolobium tomentosum Guillemin ex Benth. & 8 & $\mathrm{x}$ & & $\mathrm{x}$ & $\mathrm{x}$ & $\mathrm{x}$ & $\mathrm{x}$ & $\mathrm{x}$ & $\mathrm{x}$ & $\mathrm{x}$ & $\mathrm{x}$ & $\mathrm{x}$ & $\mathrm{x}$ \\
\hline & Lonchocarpus guillemineanus (Tul.) Malme & 7 & $\mathrm{x}$ & $\mathrm{x}$ & $\mathrm{x}$ & $\mathrm{x}$ & & $\mathrm{x}$ & & & $\mathrm{x}$ & $\mathrm{x}$ & $\mathrm{x}$ & $\mathrm{x}$ \\
\hline & Lonchocarpus muehlbergianus Hassl. & 1 & $\mathrm{x}$ & $\mathrm{x}$ & $\mathrm{x}$ & $\mathrm{x}$ & & & $\mathrm{x}$ & $\mathrm{x}$ & & $\mathrm{x}$ & $\mathrm{x}$ & \\
\hline & Machaerium villosum Vogel & 2 & $\mathrm{x}$ & $\mathrm{x}$ & & $\mathrm{x}$ & & & & & & & $\mathrm{x}$ & \\
\hline
\end{tabular}


Apêndice 1. Continuação.

\begin{tabular}{|c|c|c|c|c|c|c|c|c|c|c|c|c|c|c|}
\hline \multirow[t]{2}{*}{ Família } & \multirow[t]{2}{*}{ Espécie } & \multirow[t]{2}{*}{$\mathrm{N}$} & \multicolumn{4}{|c|}{ Queda foliar } & \multicolumn{4}{|c|}{ Floração } & \multicolumn{4}{|c|}{ Frutificação } \\
\hline & & & 2001 & 2002 & 2003 & 2004 & 2001 & 2002 & 2003 & 2004 & 2001 & 2002 & 2003 & 2004 \\
\hline & Myroxylon peruiferum L.f. & 2 & $\mathrm{x}$ & & $\mathrm{x}$ & $\mathrm{x}$ & $\mathrm{x}$ & & $\mathrm{x}$ & $\mathrm{x}$ & $\mathrm{x}$ & & $\mathrm{x}$ & \\
\hline & Platycyamus regnellii Benth. & 2 & $\mathrm{x}$ & $\mathrm{x}$ & $\mathrm{x}$ & $\mathrm{x}$ & $\mathrm{x}$ & $\mathrm{x}$ & $\mathrm{x}$ & $\mathrm{x}$ & $\mathrm{x}$ & $\mathrm{x}$ & $\mathrm{x}$ & $\mathrm{x}$ \\
\hline & Platypodium elegans Vogel & 1 & $\mathrm{x}$ & $\mathrm{x}$ & $\mathrm{x}$ & $\mathrm{x}$ & & & & $\mathrm{x}$ & & & & $\mathrm{x}$ \\
\hline Icacinaceae & Citronella gongonha (Mart.) R. A. Howard & 1 & $\mathrm{x}$ & $\mathrm{x}$ & $\mathrm{x}$ & $\mathrm{x}$ & & $\mathrm{x}$ & & & & & & \\
\hline Lauraceae & Nectandra megapotamica (Spreng.) Mez & 7 & & & & $\mathrm{x}$ & $\mathrm{x}$ & $\mathrm{x}$ & $\mathrm{x}$ & $\mathrm{x}$ & $\mathrm{x}$ & $\mathrm{x}$ & & $\mathrm{x}$ \\
\hline \multirow[t]{2}{*}{ Lecythidaceae } & Cariniana estrellensis (Raddi.) Kuntze & 2 & $\mathrm{x}$ & & $\mathrm{x}$ & $\mathrm{x}$ & & & & $\mathrm{x}$ & & & $\mathrm{x}$ & $\mathrm{x}$ \\
\hline & Cariniana legalis (Mart.) Kuntze & 5 & $\mathrm{x}$ & & $\mathrm{x}$ & $\mathrm{x}$ & $\mathrm{x}$ & $\mathrm{x}$ & & $\mathrm{x}$ & & & $\mathrm{x}$ & $\mathrm{x}$ \\
\hline Lythraceae & Lafoensia pacari A. St.-Hil. & 1 & & & $\mathrm{x}$ & & $\mathrm{x}$ & $\mathrm{x}$ & & & & $\mathrm{x}$ & $\mathrm{x}$ & $\mathrm{x}$ \\
\hline \multirow[t]{4}{*}{ Malvaceae } & Ceiba speciosa (A. St.-Hil., A. Juss. \& Cambess.) Ravenna & 6 & $\mathrm{x}$ & $\mathrm{x}$ & $\mathrm{x}$ & $\mathrm{x}$ & $\mathrm{x}$ & $\mathrm{x}$ & $\mathrm{x}$ & $\mathrm{x}$ & $\mathrm{x}$ & & $\mathrm{x}$ & \\
\hline & Guazuma ulmifolia Lam. & 5 & $\mathrm{x}$ & $\mathrm{x}$ & $\mathrm{x}$ & $\mathrm{x}$ & $\mathrm{x}$ & $\mathrm{x}$ & & $\mathrm{x}$ & $\mathrm{x}$ & $\mathrm{x}$ & $\mathrm{x}$ & $\mathrm{x}$ \\
\hline & Luehea divaricata Mart. & 7 & $\mathrm{x}$ & & $\mathrm{x}$ & $\mathrm{x}$ & $\mathrm{x}$ & $\mathrm{x}$ & & $\mathrm{x}$ & $\mathrm{x}$ & $\mathrm{x}$ & $\mathrm{x}$ & $\mathrm{x}$ \\
\hline & Pseudobombax grandiflorum (Cav.) A. Robyns & 6 & $\mathrm{x}$ & $\mathrm{x}$ & $\mathrm{x}$ & $\mathrm{x}$ & $\mathrm{x}$ & $\mathrm{x}$ & $\mathrm{x}$ & $\mathrm{x}$ & $\mathrm{x}$ & & $\mathrm{x}$ & $\mathrm{x}$ \\
\hline \multirow[t]{7}{*}{ Meliaceae } & Cabralea canjerana (Vell.) Mart. & 4 & $\mathrm{x}$ & & $\mathrm{x}$ & $\mathrm{x}$ & & $\mathrm{x}$ & $\mathrm{x}$ & $\mathrm{x}$ & $\mathrm{x}$ & $\mathrm{x}$ & & $\mathrm{x}$ \\
\hline & Cedrela fissilis Vell. & 2 & $\mathrm{x}$ & $\mathrm{x}$ & $\mathrm{x}$ & $\mathrm{x}$ & $\mathrm{x}$ & $\mathrm{x}$ & $\mathrm{x}$ & $\mathrm{x}$ & & $\mathrm{x}$ & $\mathrm{x}$ & \\
\hline & Cedrela odorata L. & 1 & & & $\mathrm{x}$ & $\mathrm{x}$ & & & & & & & & $\mathrm{x}$ \\
\hline & Guarea guidonia (L.) Sleumer & 6 & $\mathrm{x}$ & $\mathrm{x}$ & & $\mathrm{x}$ & $\mathrm{x}$ & $\mathrm{x}$ & $\mathrm{x}$ & $\mathrm{x}$ & $\mathrm{x}$ & & & \\
\hline & Trichilia catigua A. Juss. & 1 & & $\mathrm{x}$ & & & $\mathrm{x}$ & $\mathrm{x}$ & $\mathrm{x}$ & & & & & \\
\hline & Trichilia pallida $\mathrm{Sw}$. & 3 & & $\mathrm{x}$ & $\mathrm{x}$ & $\mathrm{x}$ & & $\mathrm{x}$ & $\mathrm{x}$ & $\mathrm{x}$ & & $\mathrm{x}$ & $\mathrm{x}$ & \\
\hline & Trichilia silvatica C. DC. & 1 & & & & & $\mathrm{x}$ & $\mathrm{x}$ & $\mathrm{x}$ & $\mathrm{x}$ & $\mathrm{x}$ & $\mathrm{x}$ & $\mathrm{x}$ & \\
\hline \multirow[t]{5}{*}{ Moraceae } & Ficus dendrocida Kunth & 1 & & & & & $\mathrm{x}$ & $\mathrm{x}$ & $\mathrm{x}$ & $\mathrm{x}$ & $\mathrm{x}$ & $\mathrm{x}$ & $\mathrm{x}$ & $\mathrm{x}$ \\
\hline & Ficus enormis (Mart. ex Miq) Mart. & 2 & $\mathrm{x}$ & & $\mathrm{x}$ & $\mathrm{x}$ & $\mathrm{x}$ & $\mathrm{x}$ & $\mathrm{x}$ & $\mathrm{x}$ & $\mathrm{x}$ & $\mathrm{x}$ & $\mathrm{x}$ & $\mathrm{x}$ \\
\hline & Ficus guaranitica Chodat & 4 & $\mathrm{x}$ & & $\mathrm{x}$ & $\mathrm{x}$ & $\mathrm{x}$ & $\mathrm{x}$ & $\mathrm{x}$ & $\mathrm{x}$ & $\mathrm{x}$ & $\mathrm{x}$ & $\mathrm{x}$ & $\mathrm{x}$ \\
\hline & Ficus insipida Willd. & 5 & $\mathrm{x}$ & $\mathrm{x}$ & $\mathrm{x}$ & $\mathrm{x}$ & $\mathrm{x}$ & $\mathrm{x}$ & $\mathrm{x}$ & $\mathrm{x}$ & $\mathrm{x}$ & $\mathrm{x}$ & $\mathrm{x}$ & $\mathrm{x}$ \\
\hline & Maclura tinctoria (L.) D. Don ex Steud. & 4 & $\mathrm{x}$ & $\mathrm{x}$ & $\mathrm{x}$ & $\mathrm{x}$ & $\mathrm{x}$ & $\mathrm{x}$ & $\mathrm{x}$ & $\mathrm{x}$ & $\mathrm{x}$ & $\mathrm{x}$ & $\mathrm{x}$ & $\mathrm{x}$ \\
\hline \multirow[t]{6}{*}{ Myrtaceae } & Eugenia $\mathrm{sp.}$ & 4 & & & $\mathrm{x}$ & & $\mathrm{x}$ & & & & $\mathrm{x}$ & & & \\
\hline & Myrciaria cauliflora (Mart.) O.Berg & 1 & & & & & $\mathrm{x}$ & & & & & & & \\
\hline & Myrciaria floribunda (H. West ex Willd.) O. Berg & 3 & & & & & & & & & & & & \\
\hline & Myrciaria tenella (DC.) O. Berg. & 3 & & & $\mathrm{x}$ & $\mathrm{x}$ & & $\mathrm{x}$ & & & & & & $\mathrm{x}$ \\
\hline & Psidium cattleyanum Sabine & 1 & & & & & & & $\mathrm{x}$ & & & & & \\
\hline & Psidium guajava $\mathrm{L}$. & 4 & $\mathrm{x}$ & $\mathrm{x}$ & $\mathrm{x}$ & $\mathrm{x}$ & & & & & $\mathrm{x}$ & $\mathrm{x}$ & & \\
\hline Rhamnaceae & Rhamnidium elaeocarpum Reissek & 2 & $\mathrm{x}$ & & $\mathrm{x}$ & $\mathrm{x}$ & $\mathrm{x}$ & $\mathrm{x}$ & $\mathrm{x}$ & $\mathrm{x}$ & $\mathrm{x}$ & & & \\
\hline Rubiaceae & Rudgea jasminoides (Cham.) Müll. Arg. & 18 & $\mathrm{x}$ & & & & $\mathrm{x}$ & & $\mathrm{x}$ & & $\mathrm{x}$ & $\mathrm{x}$ & $\mathrm{x}$ & $\mathrm{x}$ \\
\hline \multirow[t]{7}{*}{ Rutaceae } & Balfourodendron riedelianum (Engl.) Engl. & 1 & $\mathrm{x}$ & & & & & & & & $\mathrm{x}$ & $\mathrm{x}$ & $\mathrm{x}$ & $\mathrm{x}$ \\
\hline & Esenbeckia febrifuga (A. St.-Hil.) A. Juss. ex Mart. & 3 & & & $\mathrm{x}$ & & $\mathrm{x}$ & $\mathrm{x}$ & $\mathrm{x}$ & $\mathrm{x}$ & $\mathrm{x}$ & $\mathrm{x}$ & $\mathrm{x}$ & $\mathrm{x}$ \\
\hline & Galipea jasminiflora (A. St.-Hil.) Engl. & 5 & $\mathrm{x}$ & & & $\mathrm{x}$ & $\mathrm{x}$ & $\mathrm{x}$ & $\mathrm{x}$ & & $\mathrm{x}$ & $\mathrm{x}$ & & $\mathrm{x}$ \\
\hline & Metrodorea nigra A. St.-Hil. & 6 & $\mathrm{x}$ & & & $\mathrm{x}$ & $\mathrm{x}$ & & & & $\mathrm{x}$ & & & \\
\hline & Pilocarpus pauciflorus A. St.-Hil. & 1 & & & & & $\mathrm{x}$ & & & & & & & \\
\hline & Zanthoxylum riedelianum Engl. & 2 & $\mathrm{x}$ & $\mathrm{x}$ & $\mathrm{x}$ & $\mathrm{x}$ & & $\mathrm{x}$ & & & & & & \\
\hline & Zanthoxylum rugosum A. St.-Hil. \& Tul. & 1 & & & & & & & & $\mathrm{x}$ & & & & \\
\hline \multirow[t]{2}{*}{ Salicaceae } & Casearia gossypiosperma Briq. & 2 & $\mathrm{x}$ & $\mathrm{x}$ & $\mathrm{x}$ & $\mathrm{x}$ & $x$ & & & & & & & \\
\hline & Casearia sylvestris Sw. & 3 & & & & $\mathrm{x}$ & $\mathrm{x}$ & & $\mathrm{x}$ & $\mathrm{x}$ & $\mathrm{x}$ & $\mathrm{x}$ & $\mathrm{x}$ & $\mathrm{x}$ \\
\hline Simaroubaceae & Picramnia sellowii Planch. & 7 & $\mathrm{x}$ & & $\mathrm{x}$ & $\mathrm{x}$ & $\mathrm{x}$ & & $\mathrm{x}$ & & $x$ & $\mathrm{x}$ & & $\mathrm{x}$ \\
\hline \multirow[t]{3}{*}{ Urticaceae } & Cecropia hololeuca Miq. & 3 & & & & & $\mathrm{x}$ & $\mathrm{x}$ & $\mathrm{x}$ & $\mathrm{x}$ & $\mathrm{x}$ & $\mathrm{x}$ & $\mathrm{x}$ & $\mathrm{x}$ \\
\hline & Cecropia pachystachya Trécul & 2 & & & & & $\mathrm{x}$ & $\mathrm{x}$ & $\mathrm{x}$ & $\mathrm{x}$ & $\mathrm{x}$ & $\mathrm{x}$ & $\mathrm{x}$ & $\mathrm{x}$ \\
\hline & Urera baccifera (L.) Gaudich. ex Wedd. & 5 & & $\mathrm{x}$ & $\mathrm{x}$ & $\mathrm{x}$ & & $\mathrm{x}$ & $\mathrm{x}$ & $\mathrm{x}$ & & $\mathrm{x}$ & $\mathrm{x}$ & $\mathrm{x}$ \\
\hline Verbenaceae & Aegiphila sellowiana Cham. & 2 & $\mathrm{x}$ & $\mathrm{x}$ & $\mathrm{x}$ & $\mathrm{x}$ & & $\mathrm{x}$ & & & $\mathrm{x}$ & & & \\
\hline Vochysiaceae & Qualea jundiahy Warm. & 1 & $\mathrm{x}$ & $\mathrm{x}$ & $\mathrm{x}$ & & & & & & & & & \\
\hline
\end{tabular}

